\title{
Minimum Power Based Relay Selection for Orthogonal Multiple Access Relay Networks
}

\author{
Ayswarya Padmanabhan ${ }^{b}$, Valtteri Tervo ${ }^{b}$, Jiguang He $^{b}$, Markku Juntti ${ }^{b}$ and Tad Matsumoto ${ }^{b \dagger}$ \\ ${ }^{b}$ Centre for Wireless Communications - Radio Technologies (CWC-RT), University of Oulu, Finland - FI-90014 \\ ${ }^{\dagger}$ School of Information Science, Japan Advanced Institute of Science and Technology (JAIST), \\ Asahi-Dai 1-1, Nomi, Ishikawa, Japan 923-1292 \\ Email: firstname.lastname@oulu.fi, matumoto@jaist.ac.jp
}

\begin{abstract}
We analyze the performance of a multi-source multihelper transmission with lossy forward (LF) relaying. In LF, estimates at the relay are encoded and forwarded to the destination for improving the reliability of the received sequence transmitted from the multiple source nodes. Unlike the conventional decodeand-forward (DF) relaying, LF sends the data even in the case where decoding is not error-free. We extend the results of the channel with multiple sources and a single helper to perform relay selection by utilizing the union of rate regions. A power minimization problem is formulated using the above strategy and solved by exploiting the successive convex approximation (SCA) technique. Numerical results are presented to show that the proposed relay selection method achieves the same performance as the exhaustive search.
\end{abstract}

\section{INTRODUCTION}

Cooperative communication has attracted a lot of attention for wireless research and is likely to be a key enabling technology for efficient future network. It provides several design flexibilities such as range extension and multi-user quality of service (QoS) management. The main reason behind the exploration of user-cooperation is the willingness to share power and computation with neighbouring nodes which can lead to the saving of overall network resources. Multiple access relay channel (MARC) considers multiple source nodes communicating to a common destination with the help of one or multiple relays [1]. The main aim of deploying relays as helpers is to facilitate the probability of successful transmission to the destination. In a MARC system, relay receives information from multiple sources, performs physical layer network coding, and forwards to destination. In this paper, we consider simple exclusive OR (XOR) network coding [2] to be performed at the relays.

Decode-and-forward (DF) is one of the most classical relaying schemes [3]. In a typical DF based relaying for MARCs, networks the relay forwards the successfully decoded information sequences only, and discards the erroneous sequences in order to avoid the error propagation [4], [5]. Several resource allocation problems have been investigated for DF based multiple access relay channel (MARC). For instance, MARC cooperative network has been investigated for orthogonal transmission in [6], [7], wherein incorrectly decoded estimates at relay are forwarded to reconstruct the information sequence at the destination. Moreover, the outage probability of MARC was formulated under the assumption of orthogonal transmission and perfect source-relay links in
[6], [7]. Thus, the power allocation and relay selection is investigated in this paper for orthogonal LF MARC scenario.

A power minimization problem considering two-source onehelper was solved in [8]. Since the rate region is not known in general for multiple helpers, we use the approach presented in [8] to perform relay selection based on the union of achievable rate regions. In this paper, the system model consists of two sources and two relays. However, the generalization to an arbitrary number of sources and relays is straightforward. All source nodes choose one relay node and XOR based network coding is employed at the chosen relay [2]. Unlike the existing DF based relaying technique, we consider a method wherein only one relay node is allowed to assist the source transmission in spite of having errors. Thus, our problem is to minimize the transmit powers of source nodes and the chosen relay node so as to ensure an error-free reception at the destination node using joint network-channel coding (JNCC) decoding. Since the problem involves nonconvex constraints, it cannot be solved directly. Therefore, we adopt SCA method [9] to solve the problem in a tractable manner. Alternatively, we also consider exhaustive search based relay selection wherein we find a relay node that requires least transmit power.

The rest of the paper is organized as follows. Section II presents the system model and the conditions for reliable transmission. In Section III, the power minimization problem is formulated for the proposed method. Following the problem formulation, in Section IV, numerical simulations are illustrated for an orthogonal transmission scenario. Finally, in Section V, we conclude the paper.

\section{SySTEM MODEL}

We consider a system with two source nodes sending independent information sequences to a destination node by availing assistance from two relay nodes for a reliable transmission. All nodes are equipped with a single transmit and receive antenna and the relay nodes operate in a half duplex mode. Furthermore, we assume that there is no cooperation among the source nodes and among the relay nodes, respectively. Let $i$ and $j$ denote the $i$ th source node and the $j$ th relay node, respectively. The set of all source nodes in the system is represented by $\mathcal{S} \triangleq\{1,2\}$ and the relay nodes are denoted by $\mathcal{H} \triangleq\{1,2\}$. A pictorial representation of our system model is provided in Fig. 1(a). 


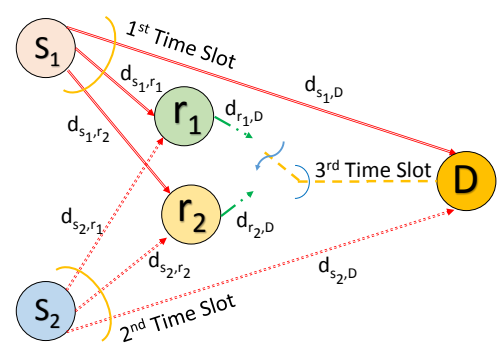

(a). Orthogonal Transmission.

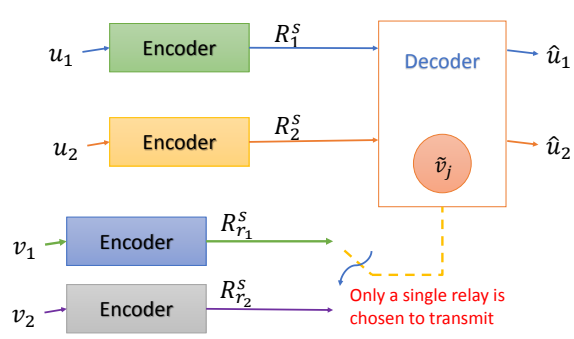

(b). Abstract model for lossless source coding.

Fig. 1. Block diagram for MARC scenario

Each source node $i, \forall i \in \mathcal{S}$ generates an independent and identically distributed (IID) binary information sequence of length $K$ bits, represented as $\mathbf{u}_{i}=\left\{u_{i}(n)\right\}_{n=1}^{K}$, which is transmitted to both relays and the destination. Upon receiving the transmitted sequences, each relay node $j$ combines the estimates $\tilde{\mathbf{u}}_{1}$ and $\tilde{\mathbf{u}}_{2}$ of both source transmissions using $\oplus$ (modulo-2) addition as $\mathbf{v}_{j}=\tilde{\mathbf{u}}_{1} \oplus \tilde{\mathbf{u}}_{2}$. Relay selection is performed such that a single relay node is chosen to forward the erroneous sequence $\tilde{\mathbf{u}}_{i} \neq \mathbf{u}_{i}$ based on the minimum total transmit power criterion. Thus, only relay node $j$, which incurs minimum power in assisting destination $D$ to decode each source sequence without errors, can act as the helper node. The transmitted symbol from source node $s_{i}$ is represented as $\mathbf{x}_{i}=\left\{x_{i}(n)\right\}_{n=1}^{N_{i}}$, where $N_{i} \in \mathbb{N}_{0}$ is the code word length corresponding to the bit sequence $\mathbf{u}_{i}$ together with the constellation used. Similarly, the relayed symbol sequence is denoted by $\mathbf{z}_{j}=\left\{z_{j}(n)\right\}_{n=1}^{N_{j}}$, where $N_{j} \in \mathbb{N}_{0}$ denotes the length of a code word. Using the above definitions, code rate of each source node $i$ and relay node $j$ are given by $\mathcal{R}_{i}^{s}=\frac{K}{N_{i}}$ and $\mathcal{R}_{r_{j}}^{s}=\frac{K}{N_{j}}$, respectively. A schematic representation of the JNCC and decoding is presented in Fig. 1(b).

Each node transmits sequentially to the destination node without any interference from the neighbouring nodes as illustrated in Fig. 1(a). It leads to a total of three time slots to complete a transmission cycle, which is organized as follows. During the first two time slots, each source $i$ encodes $\mathbf{u}_{i}$ and broadcasts the coded sequences $\mathbf{x}_{k}$ to both relay nodes and destination $D$. Thus, the received signal $\mathbf{y}_{i, j}$ at relay node $j$ and $\mathbf{y}_{i, D}$ at the destination node are given by

$$
\mathbf{y}_{i, k}=\sqrt{G_{i, k}} \sqrt{P_{i}} \mathbf{x}_{i}+\mathbf{n}_{i, k}, \forall k \in \mathcal{H} \cup\{D\}
$$

where $G_{i, j}$ and $G_{i, D}$ denote the geometric gain seen by the links between source node $i$ to relay $j$ and source node $i$ to destination $D$, respectively. The average transmit power used by source $i$ is given by $P_{i}$. The additive white Gaussian noise (AWGN) seen by relay nodes $j, \forall j \in \mathcal{H}$ and the destination node are represented as $\mathbf{n}_{i, j} \sim \mathcal{C N}\left(0, N_{0}\right)$ and $\mathbf{n}_{i, D} \sim \mathcal{C N}\left(0, N_{0}\right)$, respectively.

Following the source nodes transmission, the chosen relay node $j$ reconstructs the sequence $\mathbf{u}_{i}$ obtained from each source node $i, \forall i \in \mathcal{S}$ using the respective symbol $\mathbf{y}_{i, j}$. The received estimate of source node $i$ transmission at relay $j$ is given by $\tilde{\mathbf{u}}_{i}$. Since the relay is not interested in the original information sequence from the source nodes, the relay node simply performs a bitwise XOR of the estimated data sequences of all the sources. Without verifying the fidelity of the estimated sequence, the chosen relay node $j$ encodes the XOR'ed data symbol $\mathbf{v}_{j}$ and forwards them to destination in the respective third time slot. Now, the received symbol $\mathbf{y}_{j, D}$ at the destination from the chosen relay node $j$ is given by

$$
\mathbf{y}_{j, D}=\sqrt{G_{j, D}} \sqrt{P_{j}} \mathbf{z}_{j}+\mathbf{n}_{j, D},
$$

where $G_{j, D}$ represents the geometric gain of link between relay node $j$ to destination and $P_{j}$ denotes the average transmit power used by the relay node $j$. The AWGN noise seen by the destination for the transmission from relay node $j$ is given by $\mathbf{n}_{j, D} \sim \mathcal{C N}\left(0, N_{0}\right)$. Let $\hat{\mathbf{v}}_{j}$ be the estimate of $\mathbf{v}_{j}$ evaluated at the destination node. Now, to obtain an estimate $\hat{\mathbf{u}}_{i}$ of the original information sequence $\mathbf{u}_{i}$ from each source $i, \forall i \in \mathcal{S}$, the destination node $D$ performs JNCC decoding with all the received symbols obtained over three time slots.

Next, we analyze the conditions that ensure reliable transmission from all sources nodes $i$ to the destination node. Since the erroneous estimates of the source transmissions are allowed from each relay node, the rate region for source-torelay transmission is governed by Shannon's lossy sourcechannel separation theorem presented in [10] as

$$
\mathcal{R}_{i}^{s}\left(\mathcal{D}_{i, j}\right) \mathcal{R}_{i}^{c} \leq C\left(\widetilde{G}_{i, j} P_{i}\right), \forall i \in \mathcal{S}, j \in \mathcal{H}
$$

where $\widetilde{G}_{i, j}=\left(N_{0}\right)^{-1} G_{i, j}$ denotes the normalized link gain. The distortion $\mathcal{D}_{i j}$ is measured at the relay node $j$ for the transmission from source $s_{i}$ and $\mathcal{R}\left(\mathcal{D}_{i j}\right)=1-H\left(\mathcal{D}_{i j}\right)$ denotes the rate distortion function, where $H(\alpha)=-\alpha \log (\alpha)-(1-$ $\alpha) \log (1-\alpha)$ is the binary entropy expression. Furthermore, $C(x)=\log (1+x)$ corresponds to the link capacity between source $i$ to relay $j$ under Gaussian signaling. Since the distortion is measured by the bit flipping probability for a binary sequence, we use the Hamming distortion measure.Thus, distortion $\mathcal{D}_{i j}$ for the link between $i$ and $j$ is given by

$$
\begin{array}{r}
\mathcal{D}_{i, j}=\frac{1}{K} \sum_{n=1}^{K} d\left(u_{i}(n), \tilde{u}_{i}(n)\right) \\
d\left(u_{i}(n), \tilde{u}_{i}(n)\right)= \begin{cases}1 & \text { if } u_{i}(n) \neq \tilde{u}_{i}(n) \\
0 & \text { if } u_{i}(n)=\tilde{u}_{i}(n) .\end{cases}
\end{array}
$$

\section{Relay Selection via Union of Achievable Rate REGIONS}

It is worth noting that the capacity expression in (3) depends on the transmitted power and the code rate used by the node. Hence, there are two different approaches for the network performance maximization. 
- Maximize the total transmit code rate at source and relay nodes for fixed transmit power

- Minimize the total transmit power used by both source and relay nodes by fixing the channel codes.

We consider the latter approach. Due to the fact that multisource multi-helper rate region is not known, we use the single helper rate region derived in [11, Theorem 10.4], [8], [12] for a relay selection via the union of rate regions. The rate region for relay node $j \in \mathcal{H}$ is given by

$$
\begin{aligned}
& \mathcal{R}_{1, j}^{s} \geq H\left(\mathbf{u}_{1} \mid \mathbf{u}_{2}, \hat{\mathbf{v}}_{j}\right) \\
& \mathcal{R}_{2, j}^{s} \geq H\left(\mathbf{u}_{2} \mid \mathbf{u}_{1}, \hat{\mathbf{v}}_{j}\right) \\
\mathcal{R}_{1, j}^{s}+ & \mathcal{R}_{2, j}^{s} \geq H\left(\mathbf{u}_{1}, \mathbf{u}_{2} \mid \hat{\mathbf{v}}_{j}\right) \\
& \mathcal{R}_{r_{j}}^{s} \geq I\left(\mathbf{v}_{j} ; \hat{\mathbf{v}}_{j}\right)
\end{aligned}
$$

where $\hat{\mathbf{v}}_{j}$ is an estimate of $\mathbf{v}_{j}$ at the destination node as shown in Fig. 1(b). The mutual information (MI) and Hamming distortion between between $\mathbf{v}_{j}$ and $\hat{\mathbf{v}}_{j}$ is given as $I\left(\mathbf{v}_{j} ; \hat{\mathbf{v}}_{j}\right)$ and $\mathcal{D}_{j}$, respectively. Furthermore, $\mathcal{R}_{i, j}^{s}$ denotes the rate at which the source node $i$ transmits the sequence.

According to Shannon's source-channel separation theorem, the error probabilities of the message output from source encoders can be made arbitrarily small, if the total information rates over the four independent channels satisfy

$$
\begin{array}{r}
\mathcal{R}_{i}^{s} \mathcal{R}_{i}^{c} \leq C\left(\widetilde{G}_{i, d} P_{s_{i}}\right), \forall i \in \mathcal{S} \\
\mathcal{R}_{r_{j}}^{s} \mathcal{R}_{r_{j}}^{c} \leq C\left(\widetilde{G}_{j, d} P_{r_{j}}\right), \forall j \in \mathcal{H}
\end{array}
$$

Let us assume that a single relay node, $j$, is chosen to assist the transmissions between source and the destination node, for which, using [12], the rate region is defined as

$$
\begin{aligned}
\mathcal{R}_{i, j}^{s} & \geq H\left(\mathcal{E}_{j}\right), \forall i \in \mathcal{S} \\
\mathcal{R}_{1, j}^{s}+\mathcal{R}_{2, j}^{s} & \geq 1+H\left(\mathcal{E}_{j}\right) \\
\mathcal{R}_{r_{j}}^{s} & \geq 1-H\left(\mathcal{D}_{j}\right)
\end{aligned}
$$

where $\mathcal{E}_{j} \triangleq \mathcal{D}_{1, j} * \mathcal{D}_{2, j} * \mathcal{D}_{j}$ includes the binary convolution of the respective distortions. Since the rate region defined by each relay $j$ is independently achievable by [11, Theorem 10.4], the union of all rate regions is also achievable as given by

$$
\mathcal{R}_{i}^{s}=\min \left\{\mathcal{R}_{i, 1}^{s}, \mathcal{R}_{i, 2}^{s}\right\} .
$$

In other words, the union of achievable rate region leads to the selection of best relay node, which is used to assist the transmissions from all source nodes $i \in \mathcal{S}$ by forwarding the XOR'ed estimates to the destination.

Hence, to achieve a reliable transmission, all the conditions in (3), (4), (4), (4) and (4) must be satisfied. Upon combining the above constraints, we obtain

$$
\begin{aligned}
& 1-H\left(\mathcal{D}_{i, j}\right) \leq\left(\mathcal{R}_{i}^{c}\right)^{-1} C\left(\widetilde{G}_{i, j} P_{s_{i}}\right) \\
& 1-H\left(\mathcal{D}_{j}\right) \leq\left(\mathcal{R}_{j}^{c}\right)^{-1} C\left(\widetilde{G}_{j, d} P_{r_{j}}\right) \\
& \min \left\{H\left(\mathcal{E}_{1}\right), H\left(\mathcal{E}_{2}\right)\right\} \leq\left(\mathcal{R}_{i}^{c}\right)^{-1} C\left(\widetilde{G}_{i, d} P_{s_{i}}\right) \\
& \min \left\{1+H\left(\mathcal{E}_{1}\right), 1+H\left(\mathcal{E}_{2}\right)\right\} \leq\left(\mathcal{R}_{1}^{c}\right)^{-1} C\left(\widetilde{G}_{1, d} P_{s_{1}}\right) \\
&+\left(\mathcal{R}_{2}^{c}\right)^{-1} C\left(\widetilde{G}_{2, d} P_{s_{2}}\right) .
\end{aligned}
$$

Now, by using the above conditions, the minimum sum power formulation is given by

$$
\begin{aligned}
& \underset{\mathbf{P}, \mathbf{D}}{\operatorname{minimize}} \sum_{i \in \mathcal{S}} P_{s_{i}}+\sum_{j \in \mathcal{H}} P_{r_{j}} \\
& \text { subject to (5) }
\end{aligned}
$$

where $\mathbf{P}=\left[P_{s_{i}}, P_{r_{j}}\right]$ and $\mathbf{D}=\left[\mathcal{D}_{i, j}, \mathcal{D}_{j}\right] \forall i, \forall j$ are stacked vectors of transmit powers and distortions, respectively.

\section{A. Solution based on Successive Convex Approximation}

The SCA technique is used to find a solution for the nonconvex problem in (5). Here, we find a suitable convex subsets to the nonconvex constraints (4c) and (4d) in (5). In order to show the nonconvexity of (4c) and (4d), let us first consider the left hand side (1.h.s.) of both (4c) and (4d), which characterize the rate region. The binary entropy function $H\left(\mathcal{E}_{j}\right)$ in the 1.h.s. of both constraints (4c) and (4d) is concave. Furthermore, using $\min \{\cdot\}$ function over two concave functions yields a concave function, thereby leading to a nonconvex set. Thus, even without the use of $\min \{\cdot\}$ function, the constraints defined by (4c) and (4d) are nonconvex, which motivates us to employ SCA technique to find a feasible solution.

For clarity, let us represent (4c) and (4d) equivalently as

$$
\begin{aligned}
\min \left\{H\left(\mathcal{E}_{1}\right), H\left(\mathcal{E}_{2}\right)\right\} & \leq A_{1} \\
\min \left\{1+H\left(\mathcal{E}_{1}\right), 1+H\left(\mathcal{E}_{2}\right)\right\} & \leq A_{2}
\end{aligned}
$$

where $A_{1}$ and $A_{2}$ represent the r.h.s. of the constraints (4c) and $(4 \mathrm{~d})$, respectively. Since the terms inside $\min \{\cdot\}$ function are not convex, we resort to the SCA technique by relaxing them with the first order Taylor approximation as

$$
\begin{aligned}
H^{(k)}\left(\mathcal{E}_{j} ; \mathcal{E}_{j}^{(k)}\right) \triangleq H\left(\mathcal{E}_{j}^{(k)}\right)+ & \hat{H}\left(\mathcal{E}_{j}^{(k)}\right) \\
& \left(\mathcal{E}_{j}-\mathcal{E}_{j}^{(k)}\right) \geq H\left(\mathcal{E}_{j}\right)
\end{aligned}
$$

where $\mathcal{E}_{j}^{(k)}$ is the fixed operating point for the SCA iteration $k$ and $\hat{H}\left(\mathcal{E}_{j}^{(k)}\right)$ denotes the gradient of $\hat{H}\left(\mathcal{E}_{j}\right)$ with respect to $\mathcal{E}_{j}$. Since $\mathcal{E}_{j}$ is the convolution of relative distortion variables, i.e., $\mathcal{D}_{i, j} * \mathcal{D}_{j}$, the gradient of $\hat{H}\left(\mathcal{E}_{j}^{(k)}\right)$ is given by

$$
\hat{H}\left(\mathcal{E}_{j}\right) \triangleq \log (2)\left[1+\log \left(\mathcal{E}_{i}^{(k)}\right)\right] \times \hat{\mathcal{E}}_{j}^{(k)}
$$

where $\hat{\mathcal{E}}_{j}^{(k)}$ corresponds to the derivative of binary convolution expression $\mathcal{D}_{i, j} * \mathcal{D}_{j}$ with respect to each of the distortion variable. It is worth noting that the approximate convex function $H^{(k)}\left(\mathcal{E}_{j} ; \mathcal{E}_{j}^{(k)}\right)$ is a linear over-estimator for the concave entropy function $H\left(\mathcal{E}_{j}\right)$. Now, considering the composite term in the 1.h.s. of $(4 \mathrm{~g})$ and $(4 \mathrm{~h})$, we have the following

$$
\begin{aligned}
\min \left\{H^{(k)}\left(\mathcal{E}_{1} ; \mathcal{E}_{1}^{(k)}\right), H^{(k)}\left(\mathcal{E}_{2} ; \mathcal{E}_{2}^{(k)}\right)\right\} & \leq A_{1} \\
\min \left\{1+H^{(k)}\left(\mathcal{E}_{1} ; \mathcal{E}_{1}^{(k)}\right), 1+H^{(k)}\left(\mathcal{E}_{2} ; \mathcal{E}_{2}^{(k)}\right)\right\} & \leq A_{1}
\end{aligned}
$$

Even after relaxing the concave function $H\left(\mathcal{E}_{j}\right)$ by the respective first order over-estimator $H^{(k)}\left(\mathcal{E}_{j} ; \mathcal{E}_{j}^{(k)}\right)$, the problem is still nonconvex due to the composite minimum function. Thus, to handle the $\min \{\cdot\}$ expression, we introduce an optimization variable $\mu$ in the problem and express (9) equivalently as

$$
\mu H^{(k)}\left(\mathcal{E}_{1} ; \mathcal{E}_{1}^{(k)}\right)+(1-\mu) H^{(k)}\left(\mathcal{E}_{2} ; \mathcal{E}_{2}^{(k)}\right) \leq A_{1}
$$




$$
\begin{aligned}
\mu\left(1+H^{(k)}\left(\mathcal{E}_{1} ; \mathcal{E}_{1}^{(k)}\right)\right)+ & (1-\mu) \\
& \left(1+H^{(k)}\left(\mathcal{E}_{2} ; \mathcal{E}_{2}^{(k)}\right)\right) \leq A_{2}
\end{aligned}
$$

together with $\mu \in[0,1]$. Depending on the choice of $\mu$, either first or second relay will be chosen. The linear interpolation for $\min \{\cdot\}$ will choose either one of the values as long as they are different. If both assume the same value, then $\mu=0.5$, thereby leading to an random selection.

The 1.h.s. of (9) and (10) involves the function in difference of convex (DC) form, thus, we again resort to the SCA technique to solve the constraint by expressing it as

$$
\begin{aligned}
& {\left[\mu+\left(H^{(k)}\left(\mathcal{E}_{1} ; \mathcal{E}_{1}^{(k)}\right)-H^{(k)}\left(\mathcal{E}_{2} ; \mathcal{E}_{2}^{(k)}\right)\right)\right]^{2}+[\mu} \\
& \left.-\left(H^{(k)}\left(\mathcal{E}_{1} ; \mathcal{E}_{1}^{(k)}\right)-H^{(k)}\left(\mathcal{E}_{2} ; \mathcal{E}_{2}^{(k)}\right)\right)\right]^{2} \geq 4 A_{1}-4 B_{1} \\
& {\left[\mu+\left(H^{(k)}\left(\mathcal{E}_{1} ; \mathcal{E}_{1}^{(k)}\right)-H^{(k)}\left(\mathcal{E}_{2} ; \mathcal{E}_{2}^{(k)}\right)\right)\right]^{2}+[\mu} \\
& \left.-\left(H^{(k)}\left(\mathcal{E}_{1} ; \mathcal{E}_{1}^{(k)}\right)-H^{(k)}\left(\mathcal{E}_{2} ; \mathcal{E}_{2}^{(k)}\right)\right)\right]^{2} \geq 4 A_{2}-4 B_{1}-4(12)
\end{aligned}
$$

where the convex term $B_{1}$ is given by

$$
B_{1} \triangleq H^{(k)}\left(\mathcal{E}_{2} ; \mathcal{E}_{2}^{(k)}\right)
$$

Let us denote $C_{1}\left(\mathcal{E}_{1}, \mathcal{E}_{2} ; \mathcal{E}_{1}^{(k)}, \mathcal{E}_{2}^{(k)}\right)=H^{(k)}\left(\mathcal{E}_{1} ; \mathcal{E}_{1}^{(k)}\right)-$ $H^{(k)}\left(\mathcal{E}_{2} ; \mathcal{E}_{2}^{(k)}\right)$. The 1.h.s. of (11) and (12) is one and the same. Due to the presence of quadratic term in the 1.h.s. of (11), the constraint defined is nonconvex, and thus we approximate the quadratic function by the first order Taylor approximation as

$$
\begin{aligned}
& \text { 1.h.s. (11) } \geq\left[\mu+C_{1}\left(\mathcal{E}_{1}, \mathcal{E}_{2} ; \mathcal{E}_{1}^{(k)}, \mathcal{E}_{2}^{(k)}\right)\right]^{2} \\
& \quad-\left[\mu^{(k)}-C_{1}\left(\mathcal{E}_{1}^{(k)}, \mathcal{E}_{2}^{(k)} ; \mathcal{E}_{1}^{(k)}, \mathcal{E}_{2}^{(k)}\right)\right]^{2} \\
& -2\left[\mu^{(k)}-C_{1}\left(\mathcal{E}_{1}^{(k)}, \mathcal{E}_{2}^{(k)} ; \mathcal{E}_{1}^{(k)}, \mathcal{E}_{2}^{(k)}\right)\right]+\left[\mu-\mu^{(k)}\right. \\
& \left.-C_{1}\left(\mathcal{E}_{1}, \mathcal{E}_{2} ; \mathcal{E}_{1}^{(k)}, \mathcal{E}_{2}^{(k)}\right)+C_{1}\left(\mathcal{E}_{1}^{(k)}, \mathcal{E}_{2}^{(k)} ; \mathcal{E}_{1}^{(k)}, \mathcal{E}_{2}^{(k)}\right)\right]
\end{aligned}
$$

Using the above approximation, the SCA sub-problem to find the minimal transmit powers for the orthogonal scenario is given by

$$
\begin{aligned}
\underset{\mathbf{P}, \mathbf{D}}{\operatorname{minimize}} & \sum_{i \in \mathcal{S}} P_{s_{i}}+\sum_{j \in \mathcal{H}} P_{r_{j}} \\
\text { subject to } & (14) \geq 4 A_{1}-4 B_{1} \\
& (14) \geq 4 A_{2}-4 B_{1}-4 \\
& (4 \mathrm{a}),(4 \mathrm{~b})
\end{aligned}
$$

Once, the problem in (15) is solved, we can obtain the appropriate $\mu$ value to choose the best relay. Upon obtaining the best relay we solve the power minimization with the selected relay by shutting down the transmission via other relay completely.

It is worth noting that the proposed iterative solution in (15) is solved until convergence. However, in each iteration, we solve a convex problem efficiently using the existing solvers [9]. An algorithmic representation of the iterative procedure is provided in Algorithm 1.

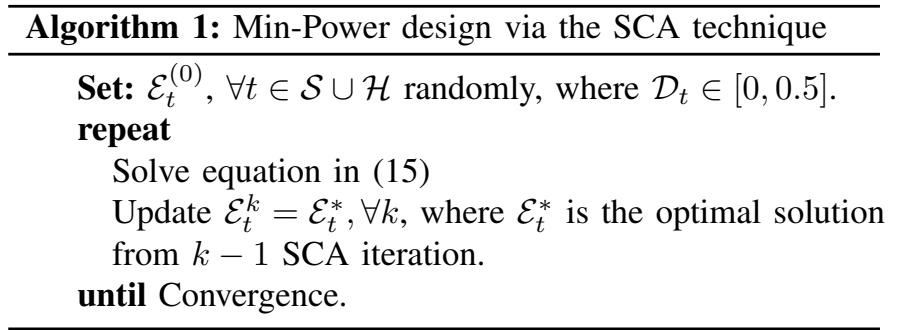

\section{Numerical Results}

In this section, we analyze the numerical results for the proposed relay selection based on orthogonal transmission scenario. The channel code rates are normalized by three, i.e., one for each source transmission and one for relay transmission, which is selected to minimize the overall transmit power. The channel code rates are fixed to $R_{i, j}=R_{r_{j}}=1$ and let $d_{i, k}$ be the distance between node $i$ and $k$, where $\forall k \in \mathcal{H} \cup\{D\}$. The path gain between nodes $i$ and $j$ is given by

$$
G_{i, k} \triangleq\left(d_{s_{1}, D} d_{i, k}^{-1}\right)^{l}
$$

where the path loss exponent is given by $l=3.52$ as in [13]. The noise power and the gain between source node $s_{1}$ and the destination are normalized to unity.

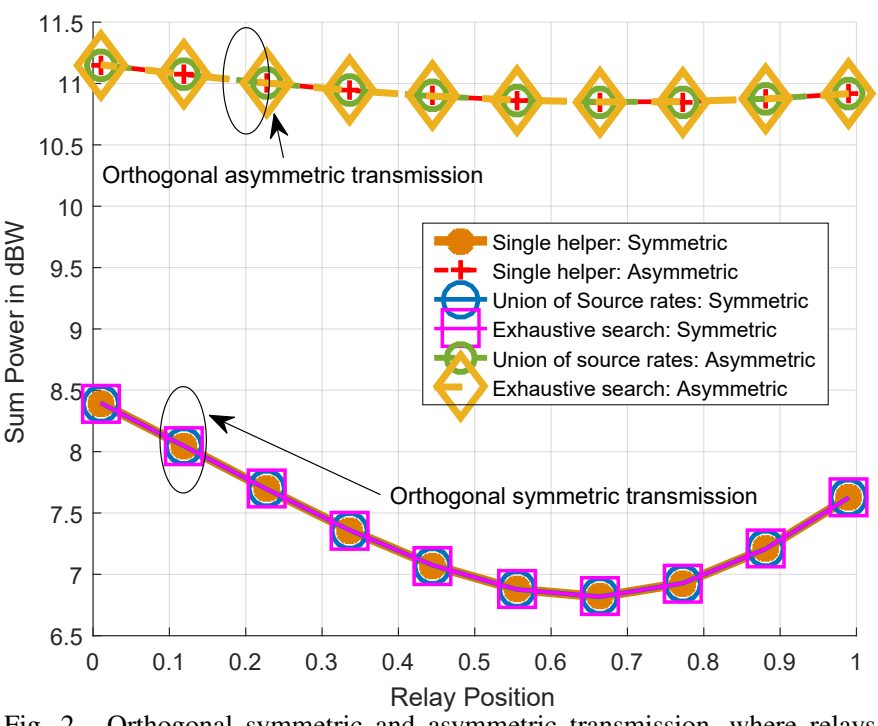

Fig. 2. Orthogonal symmetric and asymmetric transmission, where relays move in the same direction along the horizontal axis only.

For the simulation set-up, we considered two transmission topologies namely, symmetric and asymmetric, wherein for a symmetric transmission topology, we fix the source nodes and destination node at $s_{1}=(0,1), s_{2}=(0,-1)$, and $D=(1,0)$, respectively and for an asymmetric transmission topology, we fix the source nodes and destination node at $s_{1}=(0,2), s_{2}=$ $(0,-3)$, and $D=(1,0)$, respectively. However, we move the relay along horizontal axis. The figure compares sum power in $\mathrm{dBW}$ at each relay position between source and destination for the two topologies considered with an orthogonal transmission.

In Fig. 2, both relays are placed in horizontal axis and they move in same direction from source to destination. Apart from the proposed relay selection method, we also include 


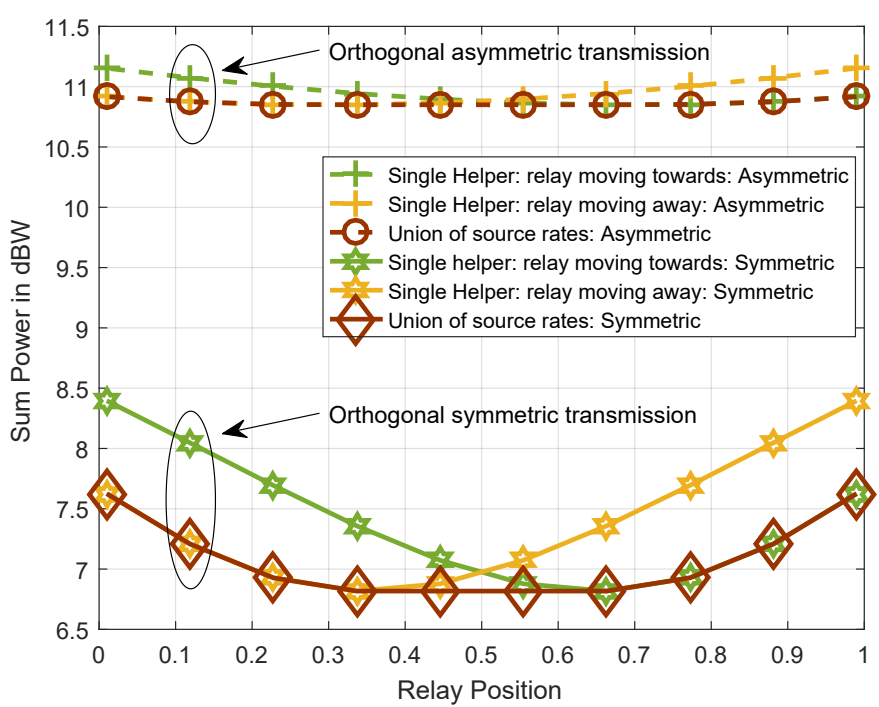

Fig. 3. Orthogonal symmetric and asymmetric transmission, where relays move in opposite direction to each other along horizontal axis only.

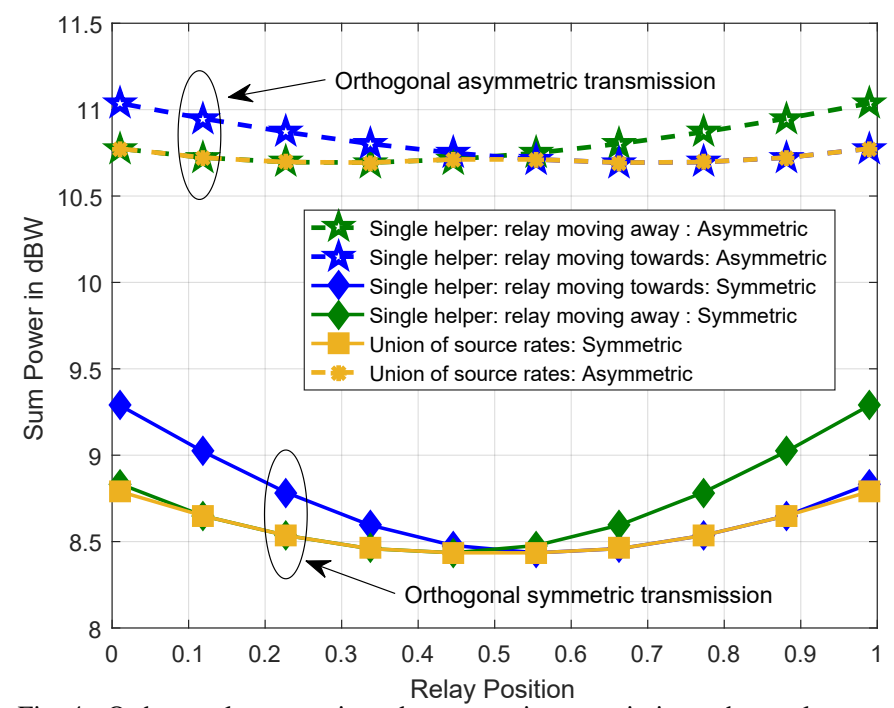

Fig. 4. Orthogonal symmetric and asymmetric transmission, where relays are fixed in vertical axis at $r_{1}=(0.5)$ and $r_{2}=(-0.5)$ and move in opposite direction to one another along horizontal axis only.

the relay selection based on exhaustive search as a benchmark scheme. We observe from the results that relay selection based on union of achievable rate region performs similar to that of single helper and exhaustive search. It is due to the reason that both the relays are equally likely as they move together and there is no gain in choosing one relay over another. However, asymmetric transmission requires more power compared to symmetric case. Note that the union of achievable rate region gives the same solution and the complexity can be reduced significantly when the number of relays grows compared to that of the exhaustive search.

In Fig. 3, two relay nodes are placed at $r_{1}=(0,0)$ and $r_{2}=$ $(0,1)$, respectively. Unlike the scenario considered in Fig. 2, relay node $r_{1}$ moves from source node to destination and relay node $r_{2}$ moves in opposite direction, i.e., from destination to source nodes. Only the horizontal axis is varied for both relay node position while keeping the vertical axis fixed. Since the relay nodes move in the opposite direction, the probability of selecting a relay node over the other depends on the union of achievable rate regions that gives the minimum compared to that of the having a single helper.

Unlike Fig. 3, we consider the case wherein relays are displaced in the vertical axis and move in opposite direction for the model presented in Fig. 4. Thus, only the horizontal axis varies for both relay node positions, while in vertical axis, relays are fixed at $r_{1}=0.5$ and $r_{2}=-0.5$, respectively. Even though initial location of relays are different, the total transmit power requirement for various relay positions behaves similar to that of the model considered in Fig. 3.

\section{CONClusion}

We evaluated the performance of multi-source using multiple helpers for the LF MARC considering relay selection utilizing union of achievable rate region. Due to the nonconvexity of the proposed power minimization problem, we employed the SCA and we have solved the original problem iteratively by solving the approximated convex sub-problems successively. We relax the problem of source coding with multiple helpers to the problem of best relay selection using the union of achievable rate regions. The performance of the proposed methods were demonstrated by numerical results and compared to the earlier results with a single helper. The relay selection utilizing union of achievable rate regions gives the same solution compared to exhaustive search.

\section{REFERENCES}

[1] G. Kramer and A. J. Van Wijngaarden, "On the white gaussian multipleaccess relay channel," in IEEE International Symposium on Information Theory, 2000. Proceedings. IEEE, 2000, p. 40.

[2] R. Ahlswede, N. Cai, S.-Y. Li, and R. W. Yeung, "Network information flow," IEEE Trans. Inf. Theory, vol. 46, no. 4, pp. 1204-1216, 2000.

[3] A. Nosratinia, T. E. Hunter, and A. Hedayat, "Cooperative communication in wireless networks," Communications Magazine, IEEE, vol. 42, no. 10 , pp. 74-80, 2004.

[4] O. Iscan and C. Hausl, "Iterative network and channel decoding for the relay channel with multiple sources," in Vehicular Technology Conference (VTC Fall), 2011 IEEE. IEEE, 2011, pp. 1-5.

[5] A. Hatefi, R. Visoz, and A. O. Berthet, "Full diversity distributed coding for the multiple access half-duplex relay channel," in 2011 International Symposium on Networking Coding. IEEE, 2011, pp. 1-6.

[6] X. Zhou, P.-S. Lu, K. Anwar, and T. Matsumoto, "Correlated sources transmission in orthogonal multiple access relay channel: Theoretical analysis and performance evaluation," IEEE Transactions on Wireless Communications, vol. 13, no. 3, pp. 1424-1435, 2014.

[7] D. H. Woldegebreal and H. Karl, "Multiple-access relay channel with network coding and non-ideal source-relay channels," in 2007 4th International Symposium on Wireless Communication Systems. IEEE, 2007, pp. 732-736.

[8] V. Tervo, P.-S. Lu, X. Zhou, M. Juntti, and T. Matsumoto, "Power allocation for orthogonal multiple access relay channel allowing intralink errors," in European Wireless Conference. IEEE, 2016.

[9] B. R. Marks and G. P. Wright, "Technical notea general inner approximation algorithm for nonconvex mathematical programs," Operations Research, vol. 26, no. 4, pp. 681-683, 1978.

[10] C. E. Shannon, "Coding theorems for a discrete source with a fidelity criterion," IRE Nat. Conv. Rec, vol. 4, no. 142-163, p. 1, 1959.

[11] A. El Gamal and Y.-H. Kim, Network information theory. Cambridge university press, 2011.

[12] P.-S. Lu, X. Zhou, and T. Matsumoto, "Outage probabilities of orthogonal multiple-access relaying techniques with imperfect source-relay links," IEEE Transactions on Wireless Communications, vol. 14, no. 4, pp. 2269-2280, 2015.

[13] R. Youssef and A. G. I. Amat, "Distributed serially concatenated codes for multi-source cooperative relay networks," IEEE Transactions on Wireless Communications, vol. 10, no. 1, pp. 253-263, 2011. 